\title{
OBITUARY
}

\section{OSKAR THEODOR}

(1898-1987)

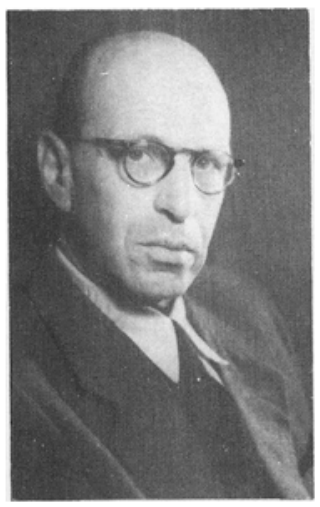

IN MEMORIAM

Oskar Theodor was born in Königsberg, on October 3, 1898. A Zionist, he came to Israel (then Palestine) at the age of 21 following one year's service as an orderly in the German army during World War I. His intention was to become a farmer. However, fairly soon, in 1921, he became involved in problems of medical entomology and became an assistant in the Government of Palestine Department of Health. In 1923 he was transferred to the Malaria Research Unit in Haifa, and in 1925 Theodor became an assistant in the Department of Parasitology of The Hebrew University of Jerusalem, where he remained throughout his entire prolific career.

Theodor left Jerusalem to complete his formal education at the University of Königsberg, where he received his Ph.D. in entomology in 1928. During the years 1942-45 he again left Jerusalem as a volunteer in the British Army and served as an officer in antimalarial campaigns in Egypt and Greece. Theodor traveled extensively for short periods, mostly as a leading authority on sandflies and leishmaniasis, to Italy, Greece, Malta, Iraq, Iran, Kenya, Ethiopia and Brazil.

Theodor was a keen naturalist with a wide knowledge of general biology. He was an ardent worker in the field as well as in the laboratory, and this versatility enabled him to carry out excellent field work on health problems as well as extensive applied and basic research. His thoroughness and persistence were remarkable even for a scientist with German training. This combination of traits was evident in the very impressive scientific work which made Theodor a leading figure in medical entomology.

Professor Theodor received wide recognition for his pioneering research on leishmaniasis. The studies which were carried out for 15 years in cooperation with the late Saul Adler, started with the elucidation of some basic facts on the life cycle and transmission of Leishmania tropica. It is difficult now, in retrospect, to fathom the void when Adler and Theodor first entered this field. For example, one of their first undertakings was to prove that Phlebotomus papatasi was the natural vector of $L$. tropica in man. This was not at all clear, since in previous investigations 
(Laveran and Franchini, 1913, 1916; Fantham and Porter, 1914, 1915) mice were infected with Herpetomonas parasites of various insects. Adler and Theodor showed (1926), using a serological test, that Herpetomonas papatasi and $L$. tropica were synonyms and that $P$. papatasi was the natural carrier of the disease.

In another paper in the same year, Theodor and Adler described new characters for the classification of female sandflies: the pharynx, buccal cavity and spermatheca. These organs came to serve as the main diagnostic characters for the definition of a species. The wide scope of their studies included the anatomy and biology of Phlebotomus spp.; the systematics of sandflies; the epidemiology and transmission of leishmaniasis; reservoir animals; vector specificity and others. All these subjects were largely terra incognita, and Adler's and Theodor's findings were a major contribution to the present knowledge on leishmaniasis.

In 1931, with publication of his new method for classifying Phlebotomus, Theodor was recognized as a world authority on the subject. In the years following, he published works on the systematics of sandflies from various regions of Asia, Africa and America. Theodor also became a leading authority on the systematics of the flies of the group Pupipara, parasitic on bats and other animals. As with sandflies, it began with the study of the fauna in Israel and later became global in scope. This work on the Streblidae, Nycteribiidae and Hippoboscidae included new keys for classification, aspects of phylogeny, zoogeography and host specificity. In the course of these studies, Theodor also described new genera and new species, which were reported in many publications. Outstanding among them is the voluminous catalogue of the Rothschild Collection of Nycteribiidae (1967), which was published by the Trustees of the British Museum.

It is impossible to cover here all the aspects of Theodor's work, but two contributions to the study of medical entomology in Israel ought to be mentioned. These are the research on ticks and transmission of relapsing fever with Adler and Schieber; and the survey of ectoparasites of mammals and birds with $M$. Costa.

After retirement, Theodor concentrated on the systematics of two non-parasitic families of Diptera, Asilidae and Bombyllidae. Interested in these flies for about 40 years, he had observed them in the field and collected the flies and the relevant literature. However, with his strict sense of propriety, he did not indulge in this work until after retirement, since the subject was outside the field of parasitology. Theodor completed this work only a few months before his death on November 17,1987 , at the age of 89 , and thereby added three beautiful monographs to the series on the local fauna published by the Israel Academy of Science.

Theodor's wife, Grete, died in 1980 . He is survived by two sons, Emanuel and Daniel, and grandchildren.

Yosef Schlein

Dept. of Parasitology

The Hebrew University of Jerusalem

Jerusalem, Israel 